\title{
Graph Layout
}

National Cancer Institute

\section{Source}

National Cancer Institute. Graph Layout. NCI Thesaurus. Code C85851.

A pictorial representation of a graph on plane, for visualization of certain properties of the graph or of the data modeled by the graph. 